\title{
Total correlations of the diagonal ensemble herald the many-body localization transition
}

\author{
J. Goold, ${ }^{1, *}$ C. Gogolin,,${ }^{2,3, \dagger}$ S. R. Clark, ${ }^{4,5,6, \dagger}$ J. Eisert, ${ }^{7, \S}$ A. Scardicchio, ${ }^{1,8, \|}$ and A. Silva ${ }^{1,9, \rrbracket}$ \\ ${ }^{1}$ The Abdus Salam International Centre for Theoretical Physics (ICTP), Strada Costiera 11, 34151 Trieste, Italy \\ ${ }^{2}$ ICFO-The Institute of Photonic Sciences, Mediterranean Technology Park, 08860 Castelldefels (Barcelona), Spain \\ ${ }^{3}$ Max-Planck-Institut für Quantenoptik, Hans-Kopfermann-Straße 1, 85748 Garching, Germany \\ ${ }^{4}$ Department of Physics, Oxford University, Clarendon Laboratory, Parks Road, Oxford OX1 3PU, United Kingdom \\ ${ }^{5}$ Department of Physics, University of Bath, Claverton Down, Bath BA2 7AY, United Kingdom \\ ${ }^{6}$ Max Planck Institute for the Structure and Dynamics of Matter, 22761 Hamburg, Germany \\ ${ }^{7}$ Dahlem Center for Complex Quantum Systems, Freie Universität Berlin, 14195 Berlin, Germany \\ ${ }^{8}$ INFN, Sezione di Trieste, Via Valerio 2, 34126 Trieste, Italy \\ ${ }^{9}$ SISSA-International School for Advanced Studies, via Bonomea, 265, 34136 Trieste, Italy
}

(Received 3 June 2015; published 5 November 2015)

\begin{abstract}
The intriguing phenomenon of many-body localization (MBL) has attracted significant interest recently, but a complete characterization is still lacking. In this work we introduce the total correlations, a concept from quantum information theory capturing multipartite correlations, to the study of this phenomenon. We demonstrate that the total correlations of the diagonal ensemble provides a meaningful diagnostic tool to pin-down, probe, and better understand the MBL transition and ergodicity breaking in quantum systems. In particular, we show that the total correlations has sublinear dependence on the system size in delocalized, ergodic phases, whereas we find that it scales extensively in the localized phase developing a pronounced peak at the transition. We exemplify the power of our approach by means of an exact diagonalization study of a Heisenberg spin chain in a disordered field. By a finite size scaling analysis of the peak position and crossover point from log to linear scaling we collect evidence that ergodicity is broken before the MBL transition in this model.
\end{abstract}

DOI: 10.1103/PhysRevB.92.180202

PACS number(s): 05.30.-d, 03.65.Ud, 73.22.Gk, 75.10.Pq

The simple paradigmatic model of a particle hopping on a lattice in the presence of disorder significantly advanced our understanding of condensed matter systems. It led to the insight that a static disordered potential can lead to a complete absence of diffusion and hence conductance in an isolated quantum system. This is known as Anderson localization following its inception by Anderson [1] more than half a century ago [2]. The original formulation focused primarily on noninteracting systems and in the years following Anderson's work a complete picture was formed: It is now known that noninteracting systems in one and two dimensions are localized for arbitrary disorder [3,4]. Anderson also conjectured that a closed system of interacting particles with sufficiently strong disorder would likewise localize and fail to equilibrate. This conjecture was only recently put on a firmer theoretical footing in a seminal work by Basko, Aleiner, and Altshuler [5]. This has led to a surge in interest in this phenomenon now known as many-body localization (MBL).

The concept of MBL has been confirmed by a number of studies [6-14], demonstrating that interacting systems can display a dynamical phase transition between a so called ergodic and a many-body localized phase. The MBL phase is characterized by robust states protected by the extensively many (approximately) local integrals of motion which emerge [15-19]. Many features of this MBL phase have since been

\footnotetext{
*jgoold@ictp.it

†publications@cgogolin.de

${ }^{\ddagger}$ s.r.clark@bath.ac.uk

$\S$ jense@ physik.fu-berlin.de

" ascardic@ictp.it

"asilva@sissa.it
}

explored. For instance, it has been shown that in the MBL phase energy eigenstates typically have low entanglement entropy with respect to any bipartition, i.e., satisfy what is called an area law [20-22]. This is in stark contrast to generic ergodic phases in which the entanglement entropy of eigenstates in the bulk of the spectrum exhibits an extensive volume law scaling. For an initial pure product state, it has also been observed that in many-body localized systems, bipartite entanglement between two sectors of the system grows only logarithmically in time [10,23-29] until an extensive value is reached. This differs notably from the usual power-law growth in ergodic systems, but also with the noninteracting case, in which a saturation to a constant is observed. At the same time, many features of MBL are still unexplored and their broader connections unknown.

In this work we go significantly beyond the previous approaches by applying a powerful and sensitive correlation measure to pin down and study the MBL transition. Our focus is on the time-averaged, dephased states that emerge from product initial states once the hopping part of the Hamiltonian is abruptly switched on. While fingerprints of the MBL transition are expected in the correlations of this dephased state, their utility depends strongly on the type of correlations considered. While the behavior of bipartite entanglement is a commonly used tool for characterization of phases by the condensed matter community, we go beyond this by employing a multipartite correlation measure for mixed states. Quantum information theory classifies correlations in quantum states as classical correlations, entanglement, quantum correlations, and total correlations, all of which have distinct physical interpretations and expose subtly different properties [30,31]. Since we expect the inherently multipartite nature of correlations to play a role in the MBL transition, we 
argue that the total correlations of the dephased state is both a meaningful and insightful quantity to investigate it.

Based on a precise condition for ergodicity, we show that the total correlations in the dephased state exhibits a different scaling with the system size in ergodic and nonergodic phases. In particular, in the disordered Heisenberg spin- $1 / 2$ chain studied we find that the total correlations grow only logarithmically with the system size in the ergodic phase, while in the MBL phase the growth is linear. Studying via exact diagonalization the total correlations averaged over disorder realizations and pure product initial states, we show that in the crossover region between these two behaviors it develops a pronounced peak with a power-law decay with the disorder strength on either side, a key signature to identify and characterize the transition. As a side remark, no peak is visible if instead of the total correlations we use the mutual information, a measure of bipartite correlations, between the left and right half of the system (see also Fig. 7 in the Supplemental Material [32]), demonstrating that multipartite correlations play an important role.

Additionally, our study connects the problem of MBL with recent research on equilibration in coherently evolving quantum systems. In the past decade this topic has seen an unprecedented revival of interest mainly due to spectacular experimental advances in cold atomic physics [33,34]. In this platform, the coherent dynamics can be followed over long time scales. In fact, there is evidence for an experimental realization of an MBL phase using cold atoms on optical lattices [35], adding further relevance to the work here.

Total correlations. The MBL transition has been investigated with a variety of tools, from transport coefficients to level statistics. A diagnostic tool to capture real space correlations in quantum states is the growth of the entanglement entropy in the evolution of product initial states [24,27]. Here, in view of the multipartite nature of correlations in interacting many-body systems, we sharpen this approach by employing the total correlations $T[30,31,36]$. In order to define $T$, we first introduce the relative entropy between two states $\rho$ and $\sigma$ defined by $S(\rho \| \sigma):=-\operatorname{tr}\left(\rho \log _{2} \sigma\right)-S(\rho)$, where $S(\rho):=-\operatorname{tr}\left(\rho \log _{2} \rho\right)$ is the von Neumann entropy. It is the quantum analog of the Kullback-Leibler divergence and a very stringent measure of the distinguishability of two quantum states [37] via a result known as quantum Stein's lemma. While not itself a metric, it still upper bounds the trace distance via Pinsker's inequality $S(\rho \| \sigma) \geqslant\|\rho-\sigma\|_{1}^{2} / 2$ [37], which captures the optimal distinguishability of quantum states with a single measurement.

We now introduce the total correlations $T$ : Let $\mathcal{P}$ be the set of all product states of a $N$-partite quantum system, i.e., for spin systems, states of the form $\pi=\pi_{1} \otimes \pi_{2} \cdots \otimes \pi_{N}$ (and the corresponding analogs for fermionic and bosonic systems). The total correlations are then defined as the minimum relative entropy between the state and any product state, i.e.,

$$
T(\rho):=\min _{\pi \in \mathcal{P}} S(\rho \| \pi) .
$$

It turns out that the unique product state which minimizes the relative entropy in the above definition is the product of the reduced states $\rho_{m}$ obtained from $\rho$ by tracing out all sites but the $m$ th, i.e., $\pi=\otimes_{m=1}^{N} \rho_{m}$ [30]. This allows us to compute the quantity straightforwardly by making partial traces over the partitions of interest. The expression for the total correlations becomes

$$
T(\rho)=\sum_{m=1}^{N} S\left(\rho_{m}\right)-S(\rho) .
$$

It is useful to point out that for $N=2$ the total correlations is equal to the mutual information, which has the operational interpretation as the work required to erase the correlations in $\rho$ [38]. If $\rho$ is a pure bipartite state, then the mutual information is equal to twice the entanglement entropy of $\rho$, i.e, $T(\rho)=$ $S\left(\rho_{1}\right)+S\left(\rho_{2}\right)$. We note that although the total correlations defined by Eq. (2) contains a contribution from the diagonal entropy studied in [9] it also contains contributions from all marginal entropies and unlike the diagonal entropy is explicitly related to the geometric picture of correlations in the state under investigation.

Quantum ergodicity, the diagonal ensemble, and manybody localization. Leaving aside the problem of a proper definition of MBL, we take the complementary approach and start by defining a property that is a condition for rightfully calling a system ergodic. The ergodic hypothesis in classical statistical physics states that ergodic systems explore their phase space uniformly such that the infinite time average and the microcanonical average should agree (making this precise is a subtle issue [39]). In quantum mechanics the time and the microcanonical average can agree exactly only for states that are evenly weighted coherent superpositions of all eigenstates in a microcanonical subspace [40]. Hence, we require less and, informally speaking, take the standpoint that to call a system, i.e., a pair of Hamiltonian and initial state, ergodic (as opposed to many-body localized) it should explore at least a constant fraction of the available Hilbert space.

Let us now turn this intuition into a clear-cut definition. The first step is to quantify the explored Hilbert space, we will do this based on the dephased or time-averaged state $\omega$. For a fixed initial state $\rho$ and nondegenerate Hamiltonian $H$ we define

$\omega:=\sum_{n}\left|E_{n}\right\rangle\left\langle E_{n}|\rho| E_{n}\right\rangle\left\langle E_{n}\right|=\lim _{\tau \rightarrow \infty} \frac{1}{\tau} \int_{0}^{\tau} d t e^{-i t H} \rho e^{i t H}$,

where $\left|E_{n}\right\rangle$ are the eigenvectors of $H$. This is often referred to as the diagonal ensemble, as the off-diagonal elements are washed away by the time average. The dephased or time-averaged state is the unique state that maximizes the von Neumann entropy given all constants of motion [41]. If the expectation value of an observable equilibrates on average during the time evolution of a system, then the equilibrium expectation value can be computed from it $[34,40]$. What is more, under mild additional conditions on the Hamiltonian the following is true: If the inverse purity $1 / \operatorname{tr}\left(\omega^{2}\right)$ of the time averaged state, also called effective dimension and participation ratio, is high, expectation values of all sufficiently local observables equilibrate on average during the time evolution even if they were initially out of equilibrium $[33,34,42,43]$.

The effective dimension, being a measure for the number of energy eigenstates that significantly contribute to the initial state $[42,43]$, can be interpreted as a measure for the explored 
Hilbert space fraction (as can other moments of the energy level occupation distribution, like $\operatorname{tr}\left(\omega^{2 q}\right)$ for $q \in \mathbb{Z}^{+}$[44]). Instead of demanding a large effective dimension for ergodicity we only demand the weaker property that $S(\omega) \geqslant$ $\log \left[1 / \operatorname{tr}\left(\omega^{2}\right)\right]$ is large enough to call a system ergodic.

To identify a reasonable notion of being large enough we take inspiration from the theory of random states (although it is important to stress that we will not actually base any of the later calculations or numerics on Haar random states). For a fixed Hamiltonian $H$ and randomly chosen unitarily invariant initial states $\rho(0)$ from the Haar measure on a microcanonical subspace of dimension $d$ one can show [45, Eq. (B6)] (compare also Refs. [46-49]) that for some $C>0$

$$
\operatorname{Pr}\left[S(\omega) \leqslant \log _{2}(d / 2)\right] \leqslant 4 \exp \left[-C d / \log _{2}(d)^{2}\right] .
$$

That is, random states typically explore at least half of the available Hilbert space in the sense that typically $S(\omega) \geqslant$ $\log _{2}(d / 2)$.

For our condition for ergodicity we relax this fraction of $1 / 2$ to a constant fraction of the available Hilbert space. To make this meaningful we have to speak about families of systems of increasing system size $N$, specify what we mean by available Hilbert space, and describe the class of initial states. As is common in localization studies we take the subspace of dimension $d$ corresponding to a fixed filling $\eta \in[0,1]$ or magnetization $2 \eta-1$ as the available Hilbert space. We then consider initial states that are pure product states with definite local particle number or magnetization from that subspace, which can be thought of as ground states of appropriate "easy" Hamiltonians. We say that a family of such systems should be considered ergodic only if most such product initial states explore at least a constant fraction of the fixed filling/magnetization subspace in the sense that for some $\lambda>0$ it holds that $S(\omega) \geqslant \log _{2}(\lambda d)$. Note that this is less restrictive than demanding that $1 / \operatorname{tr}(\omega) \geqslant \lambda d$, as $S(\omega) \geqslant-\log \operatorname{tr}(\omega)$. For families of disordered systems we demand that the same condition is fulfilled with high probability also with respect to the disorder average.

Scaling of the total correlations. We now turn to demonstrating that the total correlations in the dephased state can be used to pin down and better understand the transition point from an ergodic to the MBL phase. The key signature we exploit is the scaling of $T(\omega)$ with the system size $N$. Inspecting Eq. (2) one might expect that the total correlations in the dephased state $T(\omega)$ should generally scale extensively with $N$, i.e, for large $N$ one should have to leading order

$$
T(\omega) \propto N,
$$

as $T(\omega)$ involves the sum $\sum_{m=1}^{N} S\left(\omega_{m}\right)$ of the $N$ subsystem entropies. Indeed, this is the behavior we find in the MBL phase of the model we consider below (see also Fig. 2 in the Supplemental Material [32]).

If a family of disordered systems is ergodic however, then for some constant $\lambda>0$, for most product initial states, and with high probability over the disorder average

$$
T(\omega) \leqslant \sum_{m=1}^{N} S\left(\omega_{m}\right)-\log _{2}(\lambda d) .
$$

For a quantum spin chain of local dimension 2 at half filling $\eta=1 / 2$ the available Hilbert space dimension is $d=\left(\begin{array}{c}N \\ N / 2\end{array}\right)=$ $N ! /\left(\frac{N}{2} !\right)^{2} \geqslant \sqrt{8 \pi} e^{-2} 2^{N} / \sqrt{N}$ and $S\left(\omega_{m}\right) \leqslant \log _{2} 2=1$, so that one finds at most the logarithmic scaling

$$
T(\omega) \leqslant \log _{2}(N) / 2-\log _{2}\left(\lambda \sqrt{8 \pi} e^{-2}\right) .
$$

This is what we observe in the ergodic phase of the model we consider. One furthermore retains a logarithmic scaling for ergodic spin-1/2 systems for all other constant fillings $\eta \in[0,1]$ if another mild condition is satisfied that can also be motivated from ergodicity, namely that for some $\lambda^{\prime}>0$

$$
\sum_{m=1}^{N} S\left(\omega_{m}\right) \leqslant N s(\eta)+\lambda^{\prime} \log _{2} N,
$$

where $s(x):=-x \log _{2}(x)-(1-x) \log _{2}(1-x)$ is the binary entropy function. Equation (8) says that the sum of the local entropies of the time-averaged state should not grow much faster than one would expect for the given filling fraction $\eta$. The generalized Stirling formula implies that

$$
\log _{2} d=\log _{2}\left(\begin{array}{c}
N \\
\eta N
\end{array}\right) \geqslant N s(\eta)-c(\eta),
$$

with $c(\eta)=2 \log _{2}(e)-\log _{2}(\eta)-\log _{2}(1-\eta)$. Inserting this and Eq. (8) into (6) yields a logarithmic scaling with the system size:

$$
T(\omega) \leqslant \lambda^{\prime} \log _{2} N-\log _{2}(\lambda)+c(\eta) .
$$

This subextensive scaling can also be understood intuitively: The transport present in ergodic systems correlates the different parts of the system to the extent that they appear, for most times during the evolution, so mixed that the distinguishability from the closest product state only grows logarithmically.

Model used for numerics. A model which is known to exhibit a crossover between an ergodic and a MBL phase is the Heisenberg spin chain with random field in the $z$ direction [7]. The Hamiltonian of this model is given by

$$
H=\sum_{i=1}^{N}\left[J\left(\sigma_{x}^{i} \sigma_{x}^{i+1}+\sigma_{y}^{i} \sigma_{y}^{i+1}\right)+J_{z} \sigma_{z}^{i} \sigma_{z}^{i+1}+h_{i} \sigma_{z}^{i}\right]
$$

where the $h_{i}$ represent identically distributed static fields on each site $i$ uniformly distributed in the interval $[-h, h]$. In what follows we adopt periodic boundary conditions and set $J_{z}=1$, so that a family of systems is completely characterized by the $X X$ type coupling constant $J$ and the disorder strength $h$. For all values of the parameters, the model conserves the total magnetization $S_{z}$ along the $z$ direction, so in the numerics we have chosen the subspace with $S_{z}=0$, also referred to as half filling, i.e, $\eta=1 / 2$. We take as our initial states all product eigenstates of the on-site part of the Hamiltonian $\sum_{i=1}^{N} \sigma_{z}^{i}$ from this subspace. We then compute for each initial state the diagonal ensemble $\omega$ and $T(\omega)$. Averaging over all such initial states and disorder realizations yields $\bar{T}(\omega)$. The numerics were performed using standard libraries for matrix diagonalization. We use 10000 disorder realizations for each disorder amplitude $|h|$ and system size $N$, except for the case of $N=16$ where 1000 realizations per point were computed. 


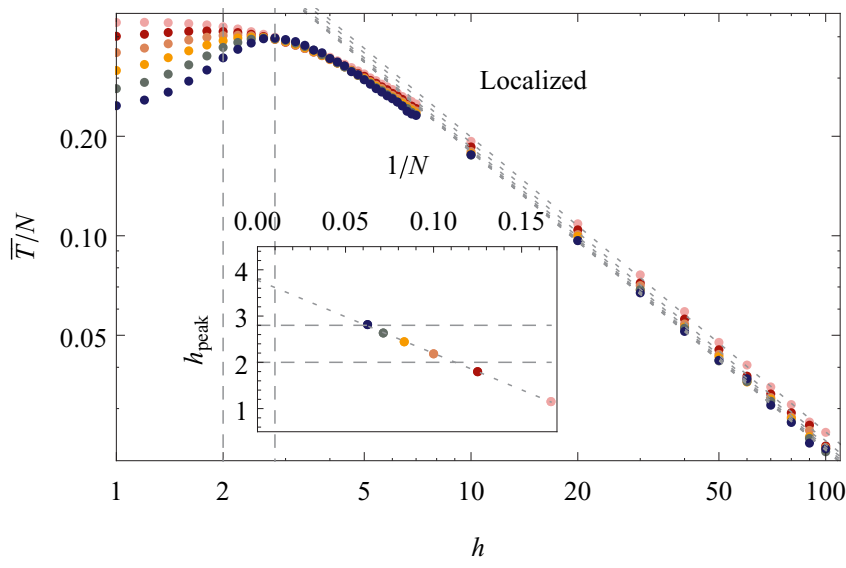

FIG. 1. (Color online) For high $h, \bar{T}$ decays as a power law. The dashed lines are fits to the data points with $h \geqslant 10$ yielding exponents of -0.9 (1) (consistent with the expectation of $h^{-1}$ corrections). The inset shows the position of the peaks from Fig. 2 (see also Fig. 6 in the Supplemental Material [32]). The extrapolated position of the peak indicates the onset of MBL at around $h=3.8$. The fact that the different $\bar{T} / N$ curves overlap for large enough $h$ indicates that $\bar{T}$ scales essentially linearly with $N$ in this regime.

Results, discussions, and conclusions. We concentrate on the case $J=J_{z}=1$. For that case the MBL transition in the model (11) was predicted to be $h_{c} \in[2,4]$ by Huse and Pal [7], with the best estimate based on energy resolved calculations being $h_{c}=3.72(6)$ [12] from an analysis of spectral statistics. Turning to the results we obtained, in all calculations performed we observe that the total correlations when plotted versus $h$ show an initial growth at low $h$ towards a maximum and then decrease monotonically at higher disorder with a power-law decay with an exponent of roughly -0.9 (2), i.e., $\bar{T} \propto N h^{-0.9(2)}$ (see Fig. 1). This is consistent with the expectation of $h^{-1}$ corrections from perturbation theory and the behavior in the noninteracting case $J_{z}=0$ (see the Supplemental Material [32]). The position of the maximum is size dependent, and can be extrapolated to be $h_{c} \approx 3.8$ in the thermodynamic limit. This is in excellent agreement with the best known approximation of the transition available in the literature [12]. In turn, by rescaling $T / N$ it appears that all curves collapse onto a single master curve for $h>h_{c}$ (see Fig. 2 in the Supplemental Material [32]). Since on qualitative grounds we expect the many-body localized phase to be characterized by (i) linear scaling $T \propto N$ and (ii) shrinking localization length as $h$ increases, it is natural to identify the MBL transition with the peak (see the inset of Fig. 1). The analysis for $h<h_{c}$ is more complex: scaling and data collapse for $T / \log (N)$, expected in an ergodic phase, are observed only for $h<2.6(2)$ (see Figs. 2 and 3). For low disorder (up until $h \approx 2.0$ for the system sizes we can access), we see a powerlaw increase of $T$ with $h$ (see Fig. 2) with an exponent of about 2.7(2). While for $h<2$ the system is definitely in an ergodic phase (compare Fig. 3 in the Supplemental Material [32]), the analysis resented in Fig. 3 suggest that ergodicity is is broken only around $h=2.6(2)$. This is consistent with an intermediate extended yet nonergodic phase [44,50] before full MBL sets in. Due to the small system size finite size effects cannot be completely ruled out. Nevertheless, recent work has demonstrated

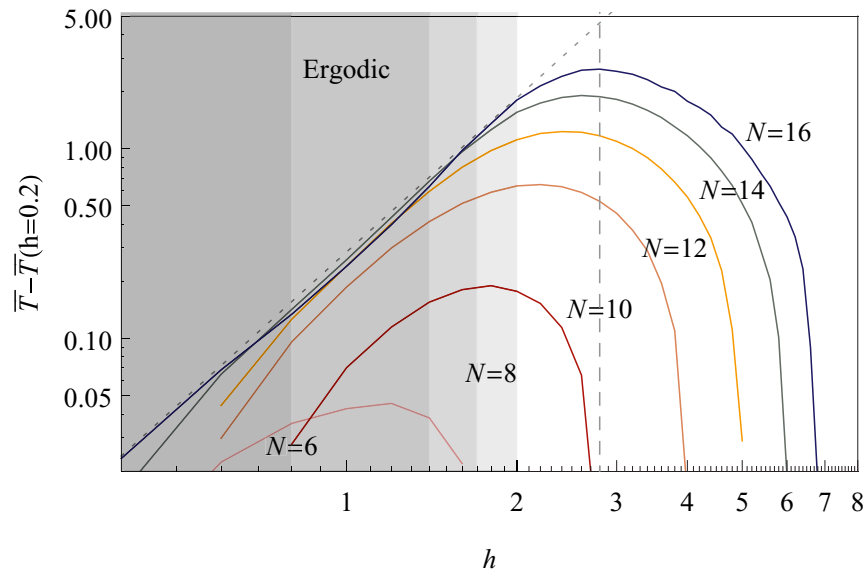

FIG. 2. (Color online) Subtracting the values of $\bar{T}$ for $h=0.2$ (which is just outside the integrable region around $h=0$ ) from $\bar{T}$ one can see that for increasing system sizes the individual curves fall on top of each other in an increasingly large region of $h$ values (shaded regions) during the approach to the peak. This increase is well captured by a power law with exponent 2.7(2) (dotted line guide to the eye $\propto h^{2.7}$ ).

an intermediate level statistics [51], implying nonergodic extended states in precisely the crossover region indicated by our numerics. We believe that our work constitutes evidence of an intermediate nonergodic region before the onset of MBL.

Conclusions. The numerical simulations performed together with our analytical arguments show that the total correlations in the diagonal ensemble signal both ergodicity breaking and the MBL transition in a quite spectacular way. In standard critical systems it is known that the multipartite correlations of the system rearrange as the system is pushed across an equilibrium phase transition [52]. Undoubtedly

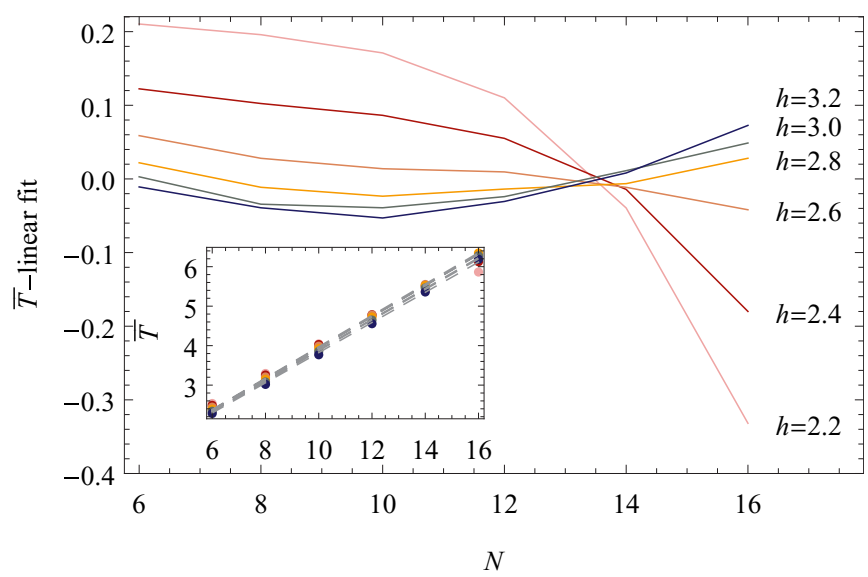

FIG. 3. (Color online) The difference of the average total correlations $\bar{T}$ and the best possible linear fit for different values of $h$. The crossover from a nearly linear scaling to a sublinear scaling clearly happens between $h=2.8$ and $h=2.4$. This result is robust against omitting data points for large or small values of $N$ and equally holds for affine fits instead of linear ones. This gives us high confidence in this result, which indicates the existence of an extended nonergodic but not yet many-body localized region. The inset shows the data before subtracting and the fits. 
the transition from an ergodic to a MBL phase is a highly nonequilibrium phenomenon which is poorly understood at present. Our approach exposes how this transition goes along with a reorganization of correlations in the dephased state via significant change in scaling with $N$. We expect this behavior to be generic and believe that the methodology outlined here is very promising to study $\mathrm{MBL}$ and ergodicity breaking phenomena in a variety of many-body quantum systems. In a follow up study we will investigate the possible multifractal nature of ergodicity breaking in a way inspired by Refs. [44,50].
Acknowledgments. This work was partially supported by the COST Action MP1209. J.E. acknowledges support by the EU (SIQS, RAQUEL, and AQUS) and the ERC (TAQ), C.G. acknowledges support by MPQ-ICFO, the Spanish Ministry Project FOQUS (FIS2013-46768-P), and the Generalitat de Catalunya (SGR 874 and SGR 875). S.R.C. acknowledges support from the ERC under the EU's Seventh Framework Programme (FP7/2007-2013)/ERC Grant Agreement No. 319286 Q-MAC. The authors acknowledge useful correspondence from M. Rigol.
[1] P. W. Anderson, Phys. Rev. 109, 1492 (1958).

[2] E. Abrahams, 50 Years of Anderson Localization (World Scientific, Singapore, 2010).

[3] P. A. Lee and T. V. Ramakrishnan, Rev. Mod. Phys. 57, 287 (1985).

[4] G. Stolz, in Entropy and the Quantum II, edited by R. Sims and D. Ueltschi (American Mathematical Society, 2010).

[5] D. M. Basko, I. L. Aleiner, and B. L. Altshuler, Ann. Phys. (Amsterdam) 321, 1126 (2006).

[6] V. Oganesyan and D. A. Huse, Phys. Rev. B. 75, 155111 (2007).

[7] A. Pal and D. A. Huse, Phys. Rev. B 82, 174411 (2010).

[8] E. Canovi, D. Rossini, R. Fazio, G. E. Santoro, and A. Silva, Phys. Rev. B. 83, 094431 (2011).

[9] A. De Luca and A. Scardicchio, Europhys. Lett. 101, 37003 (2013).

[10] J. A. Kjäll, J. H. Bardarson, and F. Pollmann, Phys. Rev. Lett. 113, 107204 (2014).

[11] R. Nandkishore and D. A. Huse, Annu. Rev. Condens. Matter Phys. 6, 15 (2015).

[12] D. J. Luitz, N. Laflorencie, and F. Alet, Phys. Rev. B. 91, 081103 (2015).

[13] Y. Bar Lev, G. Cohen, and D. R. Reichman, Phys. Rev. Lett. 114, 100601 (2015).

[14] B. Tang, D. Iyer, and M. Rigol, Phys. Rev. B 91, 161109(R) (2015).

[15] D. A. Huse, R. Nandkishore, and V. Oganesyan, Phys. Rev. B 90, 174202 (2014).

[16] J. Imbrie, arXiv:1403.7837.

[17] M. Serbyn, Z. Papić, and D. A. Abanin, Phys. Rev. Lett. 111, 127201 (2013)

[18] A. Chandran, I. H. Kim, G. Vidal, and D. A. Abanin, Phys. Rev. B 91, 085425 (2015).

[19] V. Ros, M. Müller, and A. Scardicchio, Nucl. Phys. B 891, 420 (2015).

[20] B. Bauer and C. Nayak, J. Stat. Mech. (2013) P09005.

[21] M. Friesdorf, A. H. Werner, W. Brown, V. B. Scholz, and J. Eisert, Phys. Rev. Lett. 114, 170505 (2015).

[22] J. Eisert, M. Cramer, and M. B. Plenio, Rev. Mod. Phys. 82, 277 (2010).

[23] M. Znidaric, T. Prosen, and P. Prelovsek, Phys. Rev. B. 77, 064426 (2008).

[24] J. H. Bardarson, F. Pollmann, and J. E. Moore, Phys. Rev. Lett. 109, 017202 (2012).

[25] R. Vosk and E. Altman, Phys. Rev. Lett. 110, 067204 (2013).
[26] M. Serbyn, Z. Papić, and D. A. Abanin, Phys. Rev. Lett. 110 260601 (2013).

[27] R. Vosk and E. Altman, Phys. Rev. Lett. 112, 217204 (2014).

[28] A. Nanduri, H. Kim, and D. A. Huse, Phys. Rev. B 90, 064201 (2014).

[29] M. Friesdorf, A. H. Werner, M. Goihl, J. Eisert, and W. Brown, arXiv:1412.5605.

[30] K. Modi, T. Paterek, W. Son, V. Vedral, and M. Williamson, Phys. Rev. Lett. 104, 080501 (2010).

[31] K. Modi, A. Brodutch, H. Cable, T. Paterek, and V. Vedral, Rev. Mod. Phys. 84, 1655 (2012).

[32] See Supplemental Material at http://link.aps.org/supplemental/ 10.1103/PhysRevB.92.180202 for additional information and analysis.

[33] A. Polkovnikov, K. Sengupta, A. Silva, and M. Vengalattore, Rev. Mod. Phys. 83, 863 (2011).

[34] J. Eisert, M. Friesdorf, and C. Gogolin, Nat. Phys. 11, 124 (2015).

[35] M. Schreiber et al., Science 349, 842 (2015).

[36] V. Vedral, Rev. Mod. Phys. 74, 197 (2002).

[37] K. M. R. Audenaert, Quant. Inf. Comp. 14, 31 (2014).

[38] B. Groisman, S. Popescu, and A. Winter, Phys. Rev. A. 72, 032317 (2005).

[39] J. Uffink, http://philsci-archive.pitt.edu/id/eprint/2691 (2006).

[40] J. v. Neumann, Z. Phys. 57, 30 (1929).

[41] C. Gogolin, M. P. Müller, and J. Eisert, Phys. Rev. Lett. 106, 040401 (2011).

[42] P. Reimann, Phys. Rev. Lett. 101, 190403 (2008).

[43] N. Linden, S. Popescu, A. J. Short, and A. Winter, Phys. Rev. E 79, 061103 (2009).

[44] A. De Luca, B. L. Altshuler, V. E. Kravtsov, and A. Scardicchio, Phys. Rev. Lett. 113, 046806 (2014).

[45] P. Hayden, D. Leung, P. W. Shor, and A. Winter, Commun. Math. Phys. 250, 371 (2004).

[46] E. Lubkin, J. Math. Phys. 19, 1028 (1978).

[47] S. Lloyd and H. Pagels, Ann. Phys. (NY) 188, 186 (1988).

[48] A. De Pasquale, P. Facchi, V. Giovannetti, G. Parisi, S. Pascazio, and A. Scardicchio, J. Phys. A 45, 015308 (2012).

[49] P. Facchi, U. Marzolino, G. Parisi, S. Pascazio, and A. Scardicchio, Phys. Rev. Lett. 101, 050502 (2008).

[50] B. L. Altshuler, Y. Gefen, A. Kamenev, and L. S. Levitov, Phys. Rev. Lett. 78, 2803 (1997).

[51] M. Serbyn and J. E. Moore, arXiv:1508.07293.

[52] L. Amico, R. Fazio, A. Osterloh, and V. Vedral, Rev. Mod. Phys. 80, 517 (2008). 